\title{
Micro-Ultrasound Imaging for Accuracy of Diagnosis in Clinically Significant Prostate Cancer: A Meta-Analysis
}

\begin{abstract}
Minhao Zhang ${ }^{1,2 t}$, Rong Wang ${ }^{3 t}$, Yuqing $W_{u^{1 \dagger}}$, Jibo Jing ${ }^{1 \dagger}$, Shuqiu Chen ${ }^{4}$, Guangyuan Zhang ${ }^{4}$, Bin $\mathrm{Xu}^{4 *}$, Chunhui Liu ${ }^{4 *}$ and Ming Chen ${ }^{4 *}$

${ }^{1}$ Surgical Research Center, Institute of Urology, Medical School of Southeast University, Nanjing, China, ${ }^{2}$ Department of Urology, Wuxi XiShan People's Hospital, Wuxi, China, ${ }^{3}$ Department of Urology, Affiliated Jintan Hospital of Jiangsu University, Changzhou, China, ${ }^{4}$ Department of Urology, Affiliated Zhongda Hospital of Southeast University, Nanjing, China
\end{abstract}

\section{OPEN ACCESS}

Edited by:

Zongbing You,

Tulane University, United States

Reviewed by:

Li Liu,

University of Texas Southwestern Medical Center, United States

Kaijie Wu,

First Affiliated Hospital of X'ian Jiaotong University, China

${ }^{*}$ Correspondence:

$\operatorname{Bin} X u$

njxb1982@126.com

Chunhui Liu

liuc863@126.com

Ming Chen

mingchenseu@126.com

${ }^{\dagger}$ These authors have contributed equally to this work

Specialty section:

This article was submitted to Cancer Imaging and Image-directed Interventions,

a section of the journal

Frontiers in Oncology

Received: 23 September 2019 Accepted: 20 November 2019 Published: 10 December 2019

Citation:

Zhang $M$, Wang $R$, Wu Y, Jing J,

Chen S, Zhang G, Xu B, Liu C and

Chen M (2019) Micro-Ultrasound Imaging for Accuracy of Diagnosis in Clinically Significant Prostate Cancer:

A Meta-Analysis.

Front. Oncol. 9:1368.

doi: 10.3389/fonc.2019.01368
Background: Prostate cancer is a frequently diagnosed malignant solid tumor in men. The accuracy of diagnosis is becoming increasingly important. This meta-analysis evaluated the accuracy of micro-ultrasound in the diagnosis of clinically significant prostate cancer.

Methods: We searched PubMed, Embase, Web of Science, and Cochrane Library databases to recruit studies in English. The quality assessment of diagnostic accuracy studies-2 protocol was used to evaluate the literature quality. Publication bias was analyzed using Deeks' funnel plot asymmetry test. We calculated the pooled sensitivity, specificity, positive likelihood ratio (PLR), negative likelihood ratio (NLR), diagnostic odds ratio (DOR), and 95\% confidence interval $(95 \% \mathrm{Cl}$ ) for studies of micro-ultrasound imaging for prostate cancer. The results were assessed by the summary receiveroperating characteristic curve (SROC). Ultimately, a univariable meta-regression and subgroup analysis, Fagan plot, and a likelihood matrix were conducted.

Results: A total of seven studies containing 769 patients were included in this meta-analysis. Micro-ultrasound had a pooled sensitivity, specificity, DOR, and an area under the SROC of 0.91, 0.49, 10, and 0.82, respectively. Based on these findings, micro-ultrasound has superior ability to diagnose clinically significant prostate cancer.

Conclusion: Micro-ultrasound is a more convenient and cost-effective method in realtime imaging during the biopsy procedure in detecting clinically significant prostate cancer. Although micro-ultrasound has shown promising results, more clinical data and comprehensive analysis are still needed.

Keywords: micro-ultrasound, clinically significant prostate cancer, diagnostic accuracy, biopsy, ExactVu, meta-analysis

\section{INTRODUCTION}

Prostate cancer (PCa) is a frequently diagnosed malignant solid tumor in men. It is the second leading cause of cancer deaths in the United States. In 2019, 174,650 new PCa were diagnosed, and 31,620 deaths were attributed to this disease in the United States (1). These estimated new cases and deaths are significantly higher than in 2018. In recent years, PCa has become the third 
most common type of cancer in China, and the morbidity and mortality of PCa have steadily increased (2). The prostatespecific antigen (PSA) test and digital rectal examination (DRE) are recommended for PCa screening. Magnetic resonance imaging (MRI) techniques are also used for evaluating PCa. These techniques provide unique information that is helpful to differentiate PCa from non-cancerous tissue and have been proven to improve diagnostic accuracy (3). Conventional ultrasound-based rectal systematic biopsy is insufficient, even with repeated biopsy every 6-24 months, pathological findings suggest significant differences $(4,5)$. Clinically significant $\mathrm{PCa}$ (csPCa) was considered for any Gleason sum $\geq 7$ and International Society of Urological Pathology (ISUP) grade $>2$. Rouviere et al. reported that there was no difference between systematic biopsy and MRI-targeted biopsy in the detection of PCa with ISUP grade 2 or above, but the combination of the two methods could further improve the detection rate. Multiparameter MRI examination before biopsy can improve the detection rate of csPCa, but systematic biopsy cannot be avoided. It is controversial whether multi-parameter MRI can detect more csPCa and avoid systematic biopsies (6).

The EXACTVU ${ }^{\mathrm{TM}}$ (Exact Imaging, Markham, Canada) micro-ultrasound is a novel high-resolution 29-MHz ultrasound that offers real-time biopsies targeted to suspicious areas and enables the detailed visualization of related prostate tissue characteristics, with three times greater resolution as compared with conventional ultrasound resolution (7). The ExactVu system is located in the urologist's usual procedure room. In addition to the procedure, the targeting and the entire workflow are controlled by the urologist. Micro-ultrasound also has PRIMUS (prostate risk identification using micro-ultrasound), a protocol for users to easily learn and quickly apply to help guide targeted biopsies to suspicious regions (8). The microultrasound system is a total urological solution; it operates using conventional transducers as well as high-resolution 29$\mathrm{MHz}$ biopsy transducers. Targeting is performed on the same instrument, and no MRI/fusion system is required.

Previous study indicated that micro-ultrasound may help with screening protocols by ensuring that all men with $\mathrm{PCa}$ are offered biopsy in a timely manner, while reducing the number of men without csPCa who are required to undergo the standard biopsy procedure (9). Therefore, we combined all those published evidences in a systematic manner to analyze the accuracy of diagnosis by micro-ultrasound for prostate biopsy.

\section{MATERIALS AND METHODS}

\section{Search Strategy}

This meta-analysis is based on the Preferred Reporting Items for Systematic Reviews and Meta-Analyses (PRISMA) guidelines (10). The literature research was conducted via PubMed, Web of Science, Embase, and Cochrane Library databases before July

Abbreviations: PCa, prostate cancer; csPCa, clinically significant prostate cancer; mpMRI, multiparametric magnetic resonance imaging; PRI-MUS, prostate risk identification using micro-ultrasound; PLR, positive likelihood ratio; NLR, negative likelihood ratio; DOR, diagnostic odds ratio; CI, confidence interval.
30, 2019. Keywords were "micro-ultrasound" and "prostate." Additional records were identified through the website https:// www.exactimaging.com/papers-and-publications. Two authors independently searched the databases.

\section{Criteria for Inclusion and Exclusion}

According to the standard for reporting diagnostic accuracy studies (STARD) (11), the criteria for including studies were as follows: (1) Features of lesions cannot be determined before diagnosis; (2) The group of masses was diagnosed by microultrasound; (3) The reference standard should be histopathologic diagnoses, such as biopsy or surgical pathological examination; (4) The data of four-panel (the true-positive, false-positive, falsenegative, and true-negative patients) can be obtained directly or indirectly; (5) Number of patients $\geq 15$. Exclusion criteria: (1) The reference standard is inconsistent; (2) Study design and statistical methods are improper. The published articles and the abstracts were all included in this study. The titles and abstracts of articles were independently assessed by two reviewers. The enrolled articles were evaluated and further screened by viewing the whole text.

\section{Data Extraction and Quality Assessment}

Two researchers independently collected the required data from available studies, including the name of the first author, the year of publication, distribution of population, size of sample, csPCa patients, study type, mean age, mean PSA, mean prostate volume, true-positive, false-positive, false-negative, truenegative, sensitivity, specificity, positive prediction value, and negative prediction value, if applicable.

\section{Quality Assessment and Statistical Analysis}

We used the quality assessment of diagnostic accuracy studies2 (QUADAS-2) to evaluate the quality of the literature (12). According to the bivariate mixed model (13), pooled sensitivity, specificity, positive likelihood ratio, negative likelihood ratio, and DOR were conducted to determine the accuracy of microultrasound for diagnosing $\mathrm{PCa}$. We produced a forest plot and a summary receiver-operating characteristic curve (SROC) from all of the studies. The area under the curve (AUC) was used to describe the overall accuracy as a summary of the SROC. Non-threshold heterogeneity was evaluated by the $Q$ test and $I$-squared, and $I^{2}>50 \%$ and $P<0.1$ suggested an obvious heterogeneity in terms of statistics. Meta-regression and subgroup analysis were used to identify the source of heterogeneity. Fagan plot analysis was used to assess the relationship among the pretest probability of the disease, the likelihood ratio of the diagnostic test, and posttest probability of the disease. We also generated a likelihood matrix, which is represented as a scatter plot of the positive and negative likelihood ratios. We used STATA software version 15.0 (Stata Corporation, College Station, TX, USA) and Review Manager software (RevMan, Version 5.3) to analyze the data. A $P$-value $<0.05$ suggested statistical significance. 


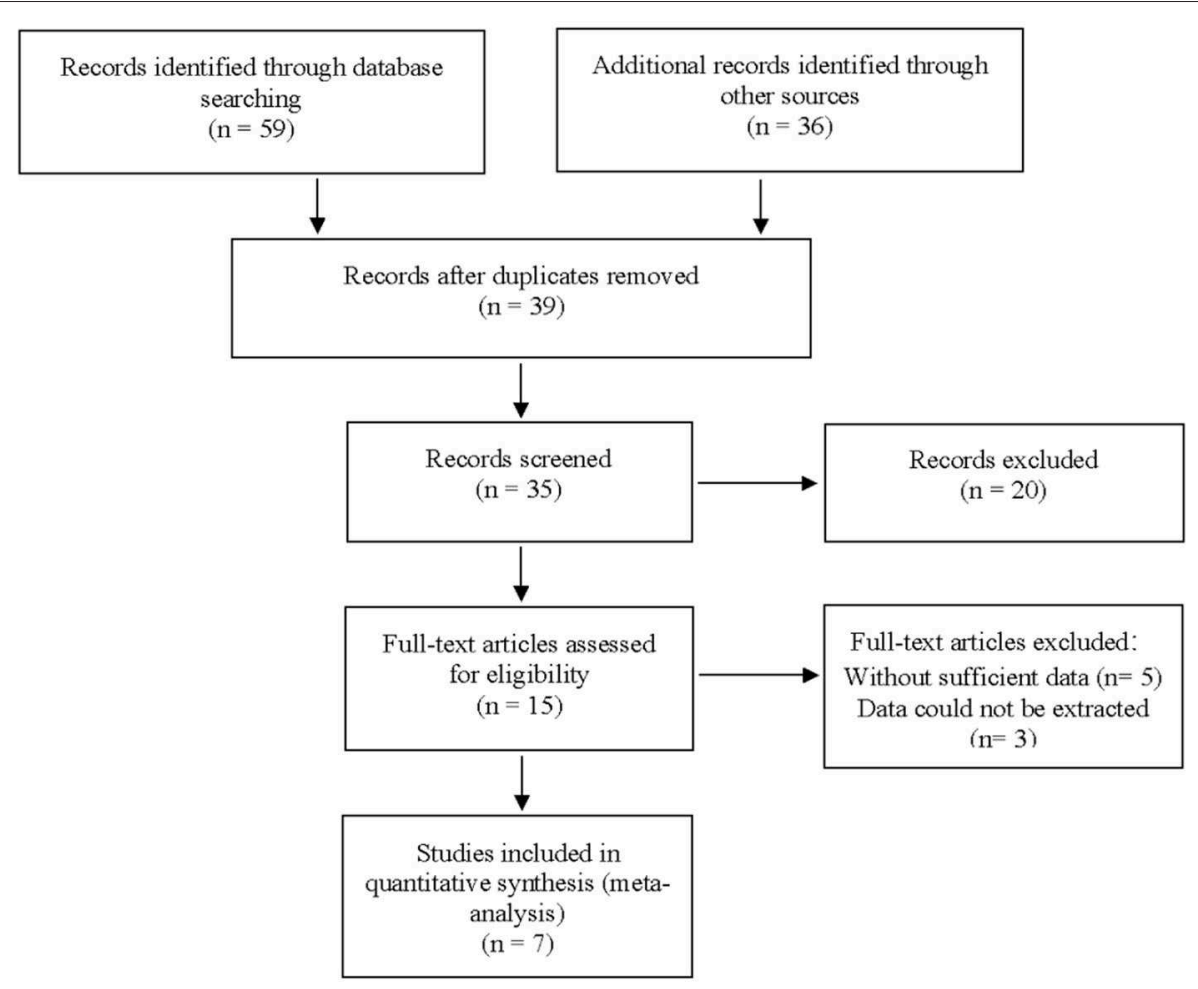

FIGURE 1 | The flowchart for the identification of eligible studies.

TABLE 1 | Baseline characteristics of included articles.

\begin{tabular}{|c|c|c|c|c|c|c|c|c|}
\hline References & Cases & Study design & Blinded & Consecutive & Mean age & $\begin{array}{l}\text { Mean PSA } \\
(\mathrm{ng} / \mathrm{ml})\end{array}$ & $\begin{array}{l}\text { Mean prostate } \\
\text { volume }(\mathrm{ml})\end{array}$ & $\begin{array}{l}\text { Reference } \\
\text { standard }\end{array}$ \\
\hline Lughezzani et al. (14) & 286 & Prospective & Yes & Yes & 64 & 9 & 57.3 & Histology \\
\hline Astobieta et al. (15) & 35 & Prospective & Yes & Yes & $N R$ & NR & NR & Histology \\
\hline Abouassaly et al. (16) & 67 & Prospective & No & Yes & 66 & 5.37 & 38 & Histology \\
\hline Chessa et al. (17) & 68 & Prospective & Yes & Yes & $N R$ & NR & NR & Histology \\
\hline Claros et al. (18) & 48 & Retrospective & No & No & 66.9 & 9.1 & 54.4 & Histology \\
\hline Luger et al. (19) & 142 & Prospective & Yes & Yes & 66 & 5.39 & NR & Histology \\
\hline Eure et al. (20) & 123 & Retrospective & Yes & Yes & NR & NR & NR & Histology \\
\hline
\end{tabular}

PSA, prostate specific antigen; NR, not reported.

\section{RESULTS}

\section{Study Characteristics}

A total of 59 articles were identified in our literature search. Thirty-nine articles were included in this systematic review after eliminating duplicate articles. According to the inclusion criteria in the study selection process, seven articles were selected for the meta-analysis (Figure 1). The baseline characteristics of the included studies are presented in Table $\mathbf{1}$. As shown in Table 2, the data related to micro-ultrasound diagnosis of csPCa are presented. The pooled sensitivity, pooled specificity, likelihood ratios, and AUROC are provided in Table 3 and Figure 2.

\section{Quality Assessment in Included Studies}

The quality of all seven available studies in our meta-analysis was evaluated based on the QUADAS-2 protocol, and the risk of bias and applicability concerns of seven studies is shown in Figure 3. In general, the quality of the included studies was considered high. Regarding the patient selection domain, Gregg's study was considered to have a high risk of bias as the included patients were not identified by pathology. Regarding the index test domain, the studies by Abouassaly et al. and Claros et al. were considered to have unclear risk because blinding was unclear. Regarding the reference standard domain, three studies had unclear risk of bias as it is uncertain whether the interpretation of the reference standard used the blind method. Regarding the flow 
TABLE 2 | Summary of results of micro-ultrasound in included studies.

\begin{tabular}{lcccc}
\hline References & \multicolumn{4}{c}{ Micro-ultrasound } \\
\cline { 2 - 5 } & TP & FP & FN & TN \\
\hline Lughezzani et al. (14) & 94 & 141 & 9 & 42 \\
Astobieta et al. (15) & 20 & 1 & 1 & 13 \\
Abouassaly et al. (16) & 21 & 29 & 7 & 10 \\
Chessa et al. (17) & 39 & 3 & 18 & 8 \\
Claros et al. (18) & 18 & 11 & 1 & 18 \\
Luger et al. (19) & 48 & 66 & 0 & 28 \\
Eure et al. (20) & 8 & 59 & 1 & 55
\end{tabular}

TP, true-positive; FP, false-positive; FN, false-negative; TN, true-negative.

TABLE 3 | Pooled sensitivity, pooled specificity, pooled likelihood rations, and AUROC of micro-ultrasound.

\begin{tabular}{lc}
\hline Pooled sensitivity & 0.91 \\
$(95 \% \mathrm{Cl})$ & $(0.79-0.97)$ \\
Pooled specificity & 0.49 \\
$(95 \% \mathrm{Cl})$ & $(0.30-0.69)$ \\
Pooled positive LR & 1.80 \\
$(95 \% \mathrm{Cl})$ & $(1.20-2.70)$ \\
Pooled negative LR & 0.18 \\
$(95 \% \mathrm{Cl})$ & $(0.07-0.50)$ \\
Pooled DOR & 10.00 \\
$(95 \% \mathrm{Cl})$ & $(3.00-35.00)$ \\
AUROC & 0.82 \\
$(95 \% \mathrm{Cl})$ & $(0.78-0.85)$ \\
\hline
\end{tabular}

AUROC, area under the summary receiver-operating characteristic curve; Cl, confidence interval.

and timing domain, the study by Abouassaly et al. was considered to have unclear risk. There was low concern for applicability with regard to the first three QUADAS-2 domains for all seven included studies.

\section{Heterogeneity Test and Subgroup Analysis}

We analyzed the sensitivity and specificity of micro-ultrasound in seven studies. A $P$-value $<0.05$ indicated that significant heterogeneity exists among these seven studies. As shown in Figure 4, the forest plots of micro-ultrasound indicated that the heterogeneity existed among the included articles. In addition, we used meta-regression analysis to evaluate various covariates from these studies, including the "whether blinding was applied," "study type," "consecutive or random," and "cases." The detailed data for the meta-regression analysis are presented in Figure 5. From the specificity results of micro-ultrasound, the covariates of "cases" were statistically significant. Thus, the results of this meta-regression analysis suggested that the sample size may be the source of potential heterogeneity.

\section{Publication Bias}

The publication bias for micro-ultrasound was determined through Deeks' funnel plot. The shape of the funnel plots was

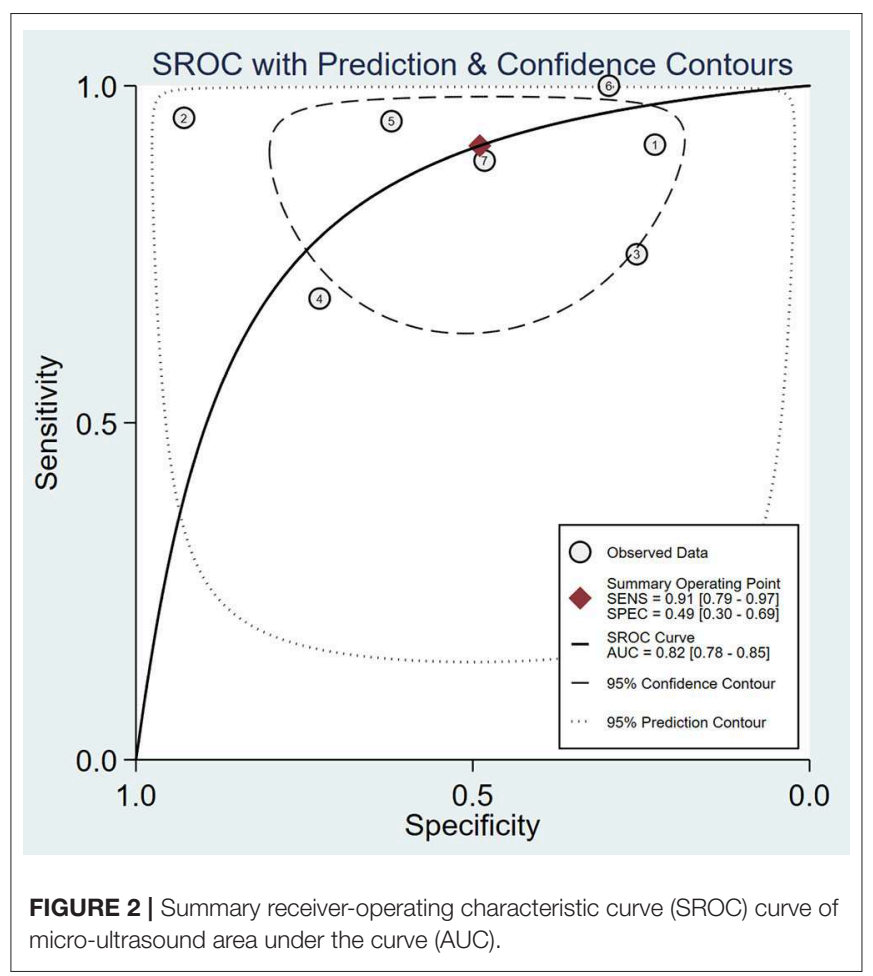

almost symmetrical, suggesting low publication bias $(P=0.41$; Figure 6).

\section{Fagan Plot Analysis and Likelihood Matrix}

Likelihood ratio and posttest probability are closely related to clinical disease. In our study, both the likelihood ratio and posttest probability were moderate (Figure 7). Given a pretest probability of $50 \%$, the positive posttest probability is $64 \%$, and the negative posttest probability is $15 \%$.

As shown in Figure 8, the summary PLR and NLR for microultrasound diagnosis of csPCa were concentrated in the right lower quadrant. This information indicates that the PLR was $<10$ and the NLR was $>0.1$.

\section{DISCUSSION}

People with suspected PCa usually need prostate biopsy first, which can result in morbidity, such as bleeding, infection, and rectal and bladder injury. Increasing the positive rate of suspected prostate lesions can significantly reduce unnecessary biopsies and complications. Previous studies demonstrated that MRI has high sensitivity and specificity $(21,22)$. The application of MRI for prostate biopsy is a usual method, but it is not recommended as an alternative for systemic biopsy at present (23). Micro-ultrasound, as a novel high-resolution imaging method for prostate biopsy, has received increasing attention. In our meta-analysis, we evaluated the diagnostic accuracy of microultrasound for csPCa. After our comprehensive and systematic literature retrieval and verification, a total of seven studies met the inclusion criteria. Micro-ultrasound's high sensitivity makes it an attractive option for guiding targeted biopsy (pooled 


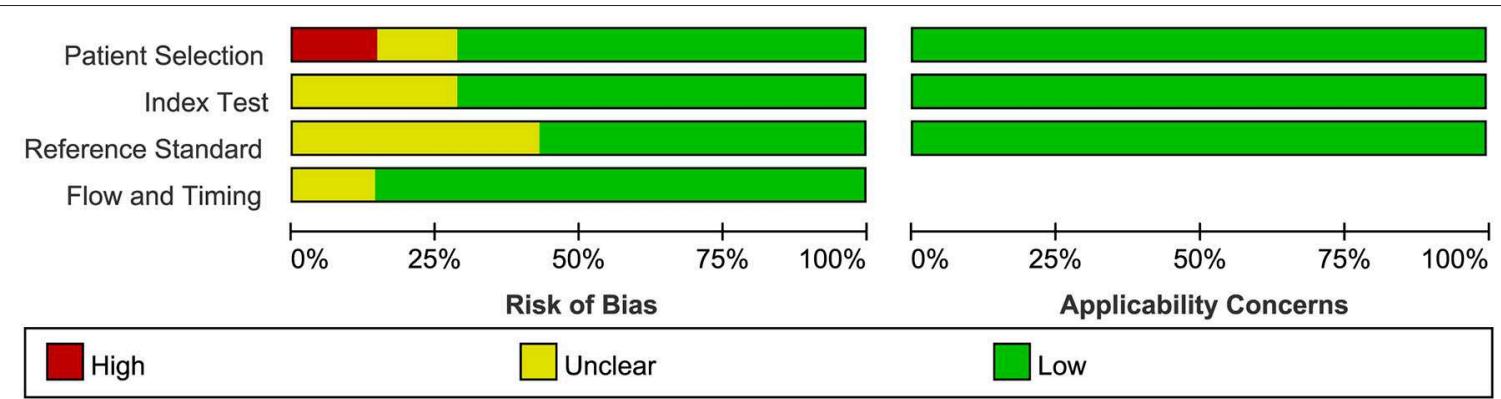

FIGURE 3 | Group bar charts show risk of bias and applicability concerns of the seven included records using quality assessment of diagnostic accuracy studies-2 (QUADAS-2).

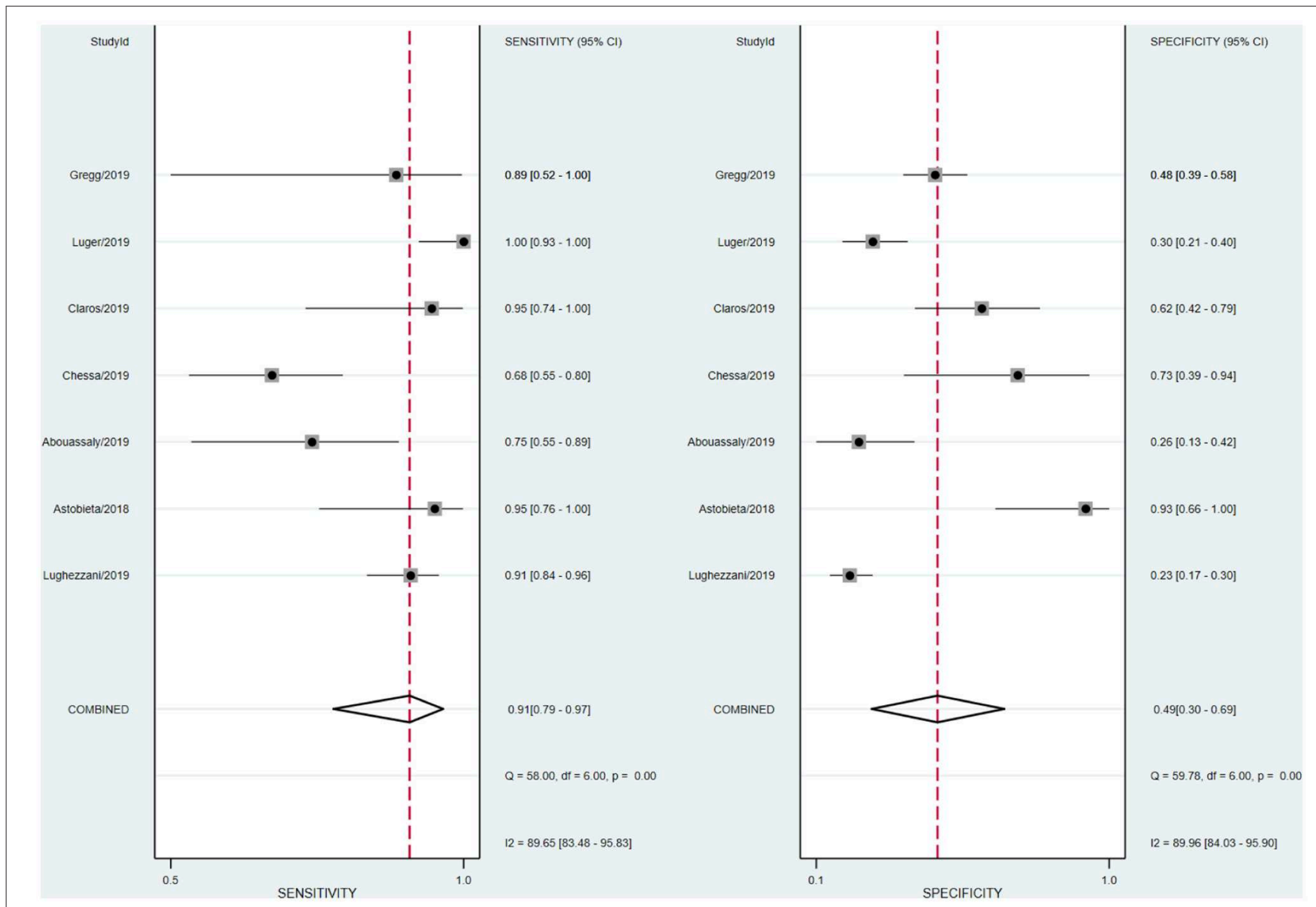

FIGURE 4 | Forest plots of sensitivity and specificity of micro-ultrasound.

sensitivity $91 \%$, pooled specificity $49 \%$ ). We calculated the DOR, a single indicator of test accuracy, and the mean DOR was 10, which demonstrates a high level of overall accuracy. In addition, we calculated the AUC of micro-ultrasound (AUROC 0.82), which indicated a high level of overall diagnostic accuracy.

The forest plot indicated that heterogeneity existed in sensitivity and specificity among the studies $\left(I^{2}>50 \%\right)$. The univariable meta-regression and subgroup analysis revealed that the covariates "cases" might be the potential source of heterogeneity with regard to specificity. In studies of microultrasound by Chessa et al. and Abouassaly et al., the sensitivity was 0.68 and 0.75 , respectively $(16,17)$. There were 57 and 28 patients in the two studies, respectively, with positive lesions among the 39 and 21, respectively, who had successful detection, leading to the lower sensitivity. In the studies written by Luger et al., Abouassaly et al., and Lughezzani et al., the specificities 

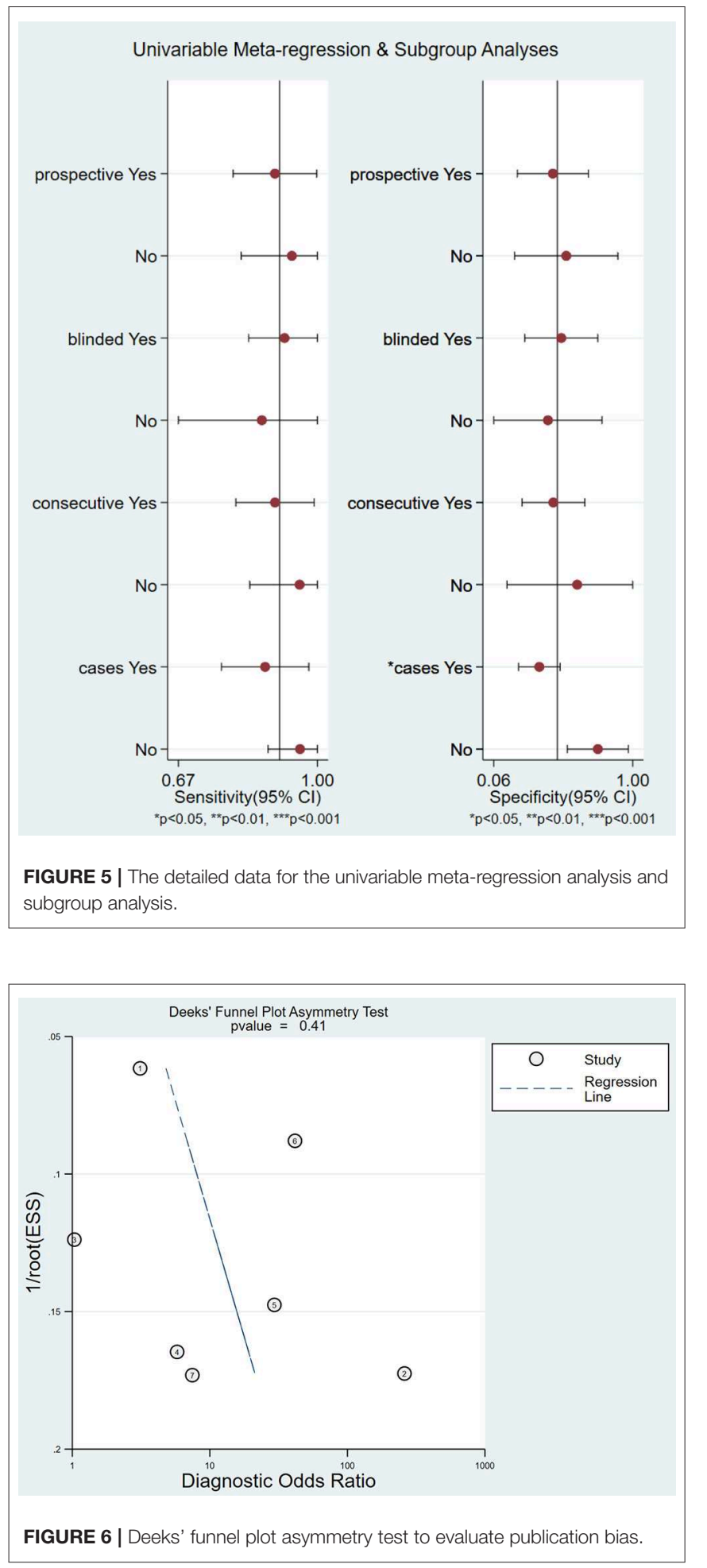

were $0.30,0.26$, and 0.23 , respectively $(14,16,19)$. Astobieta et al. (15) indicated that there were 14 patients with negative lesions among those who had successful detection, and 13 of them showed negative prediction, leading to the highest specificity of 0.93. The studies of micro-ultrasound by Claros et al. and Eure et al. showed that the specificities were 0.62 and 0.48 , respectively

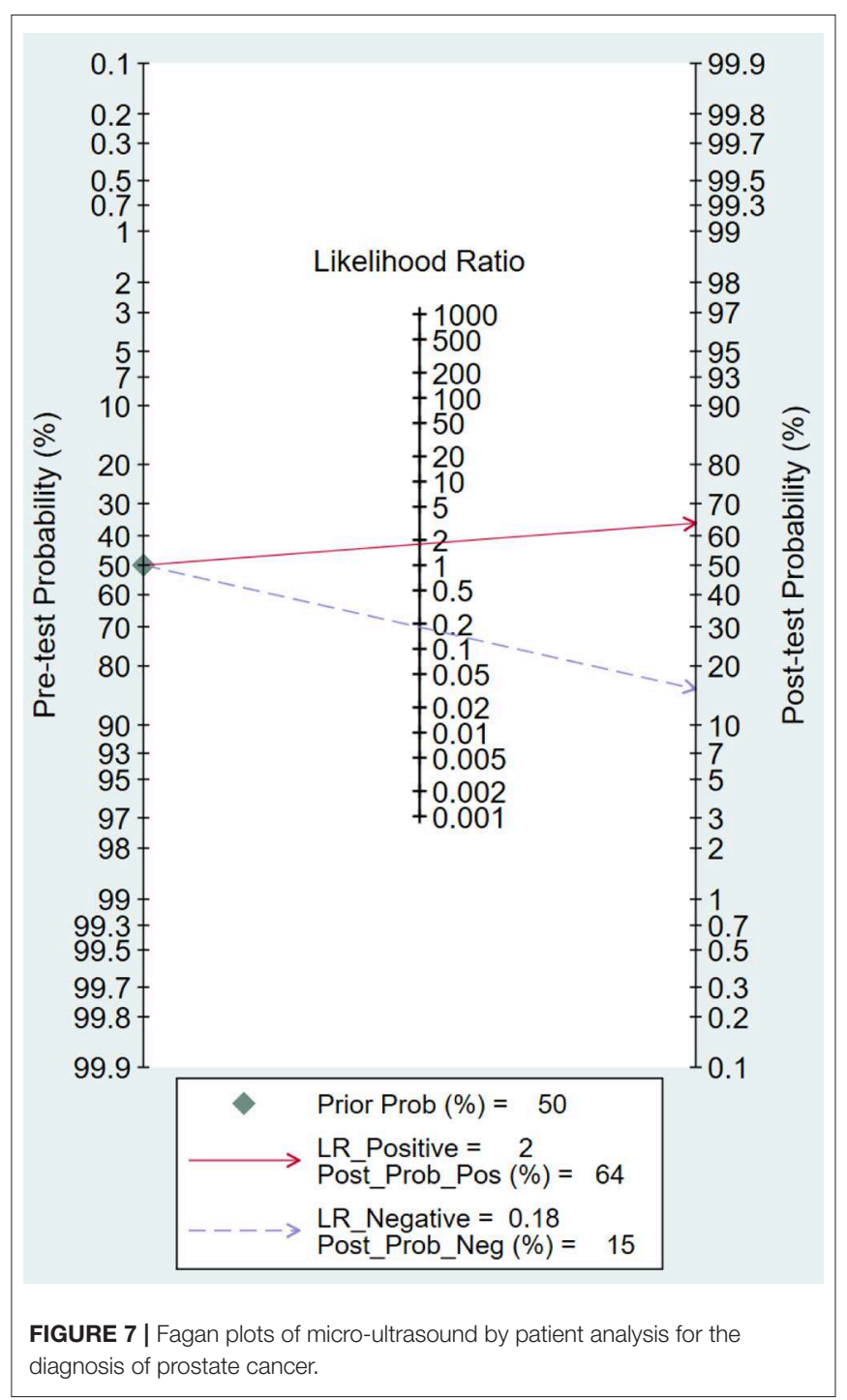

$(18,20)$. The results of our meta-analysis indicated that microultrasound had high sensitivity for detection of csPCa, but the specificity was moderate.

On the other hand, Fagan plot analysis is also important to determine the effectiveness of a diagnostic test. Fagan plot analysis indicated that micro-ultrasound has limited value in improving the diagnosis and exclusion of csPCa, and the result suggested that the use of micro-ultrasound cannot confirm or exclude malignancy.

A likelihood ratio plot was drawn to visually demonstrate that micro-ultrasound is effective in improving the accuracy of csPCa diagnosis. Epidemiological study (24) suggested that a pooled positive likelihood ratio $>10$ and pooled negative likelihood ratio $<0.1$ indicated diagnostic value for csPCa. However, all of the scatter points were clustered in the lower right quadrant. These results suggested that the use of microultrasound cannot confirm or exclude malignancy. We need to combine additional clinical data and tests for a more comprehensive analysis. 


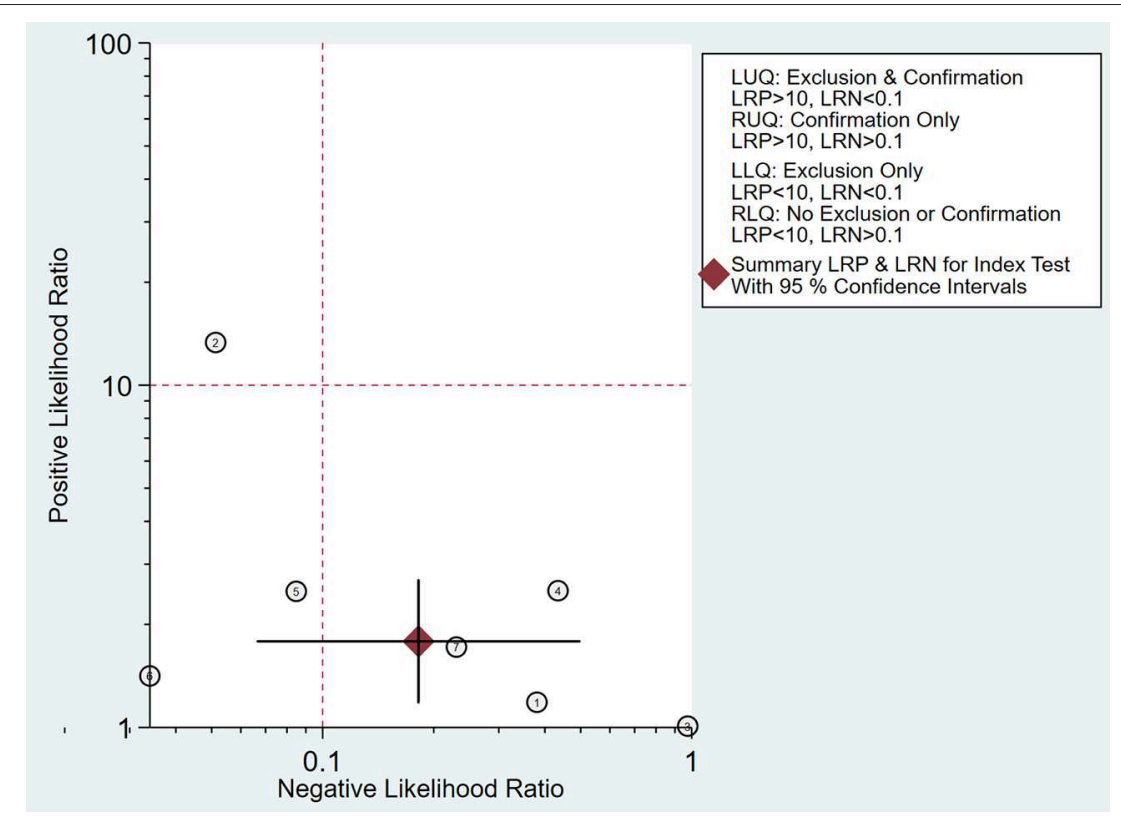

FIGURE 8 | Likelihood matrix indicates that summary positive likelihood ratio (PLR) and negative likelihood ratio (NLR) for micro-ultrasound diagnosis of clinically significant prostate cancer (csPCa) with 95\% confidence intervals are concentrated on the right lower quadrant (RLQ).

Our meta-analysis was based on data extracted from published literature. In addition, we objectively analyzed the application of micro-ultrasound in the diagnosis of csPCa by calculating the pooled sensitivity and specificity. Fagan plot and likelihood matrix were applied to evaluate the accuracy of csPCa diagnosis. Finally, we used stratified analysis to examine the results of variables in subgroups of patients. This study has several limitations. First, there may be linguistic bias in the retrieval of English literature only. Second, there is a lack of quality assessment criteria for studies. However, to address this, our study is based on strict inclusion criteria and quality evaluation criteria; the included articles are high quality after quality assessment, and the data are reliable. No meta-analysis has ever been published in this area; the analysis methods are scientific and rigorous, which we used. Third, according to these results, micro-ultrasound has high sensitivity but poor specificity. The detection ability of micro-ultrasound is strong, but the possibility of misdiagnosis is high, and additional studies are needed to obtain better application values. The low specificity of micro-ultrasound may be due to the learning curve. However, compared with single study, the results have greater efficiency and credibility. We need large-scale studies to validate the clinical application of micro-ultrasound as a diagnostic tool for csPCa.

\section{CONCLUSION}

In our meta-analysis of seven studies, micro-ultrasound is a more convenient and cost-effective method in real-time imaging during the biopsy procedure in detecting clinically significant prostate cancer. Although micro-ultrasound has promising results, more clinical data and comprehensive analysis are needed.

\section{DATA AVAILABILITY STATEMENT}

All datasets generated for this study are included in the article/supplementary material.

\section{AUTHOR CONTRIBUTIONS}

$\mathrm{MZ}, \mathrm{BX}$, and CL contributed to the conception and design of the study. RW organized the databases. YW and JJ performed the statistical analysis. MZ and YW wrote the first draft of the manuscript. SC, GZ, and MC wrote sections of the manuscript. All authors contributed to manuscript revision, read, and approved the submitted version.

\section{FUNDING}

This study was funded by The National Natural Science Foundation of China (Nos. 81872089, 81370849, 81672551, 81300472, 81070592, 81202268, and 81202034), Natural Science Foundation of Jiangsu Province (BK20161434, BL2013032, BK20150642, and BK2012336), Six talent peaks project in Jiangsu Province, Jiangsu Provincial Medical Innovation Team (CXTDA2017025, WSW-034), and Jiangsu Provincial Medical Talent (ZDRCA2016080). 


\section{REFERENCES}

1. Siegel RL, Miller KD, Jemal A. Cancer statistics, 2018. CA Cancer J Clin. (2018) 68:7-30. doi: 10.3322/caac.21442

2. Chen W, Zheng R, Baade PD, Zhang S, Zeng H, Bray F, et al. Cancer statistics in China, 2015. CA Cancer J Clin. (2016) 66:115-32. doi: 10.3322/caac.21338

3. Kim JK, Jang YJ, Cho G. Multidisciplinary functional MR imaging for prostate cancer. Korean J Radiol. (2009) 10:535-51. doi: 10.3348/kjr.2009.10.6.535

4. Al Otaibi M, Ross P, Fahmy N, Jeyaganth S, Trottier H, Sircar K, et al. Role of repeated biopsy of the prostate in predicting disease progression in patients with prostate cancer on active surveillance. Cancer. (2008) 113:28692. doi: $10.1002 / \mathrm{cncr} .23575$

5. Jain S, Loblaw A, Vesprini D, Zhang L, Kattan MW, Mamedov A, et al. Gleason upgrading with time in a large prostate cancer active surveillance cohort. $J$ Urol. (2015) 194:79-84. doi: 10.1016/j.juro.2015.01.102

6. Rouviere O, Puech P, Renard-Penna R, Claudon M, Roy C, MegeLechevallier $\mathrm{F}$, et al. Use of prostate systematic and targeted biopsy on the basis of multiparametric MRI in biopsy-naive patients (MRI-FIRST): a prospective, multicentre, paired diagnostic study. Lancet Oncol. (2019) 20:100-9. doi: 10.1016/S1470-2045(18) 30569-2

7. Rohrbach D, Wodlinger B, Wen J, Mamou J, Feleppa E. High-frequency quantitative ultrasound for imaging prostate cancer using a novel micro-ultrasound scanner. Ultrasound Med Biol. (2018) 44:1341-54. doi: 10.1016/j.ultrasmedbio.2018.02.014

8. Ghai S, Eure G, Fradet V, Hyndman ME, McGrath T, Wodlinger B, et al. Assessing cancer risk on novel $29 \mathrm{MHz}$ micro-ultrasound images of the prostate: creation of the micro-ultrasound protocol for prostate risk identification. J Urol. (2016) 196:562-9. doi: 10.1016/j.juro.2015.12.093

9. Ghai S, Van der Kwast T. Suspicious findings on micro-ultrasound imaging and early detection of prostate cancer. Urol Case Rep. (2018) 16:98-100. doi: 10.1016/j.eucr.2017.11.013

10. Panic N, Leoncini E, de Belvis G, Ricciardi W, Boccia S. Evaluation of the endorsement of the preferred reporting items for systematic reviews and meta-analysis (PRISMA) statement on the quality of published systematic review and meta-analyses. PLoS ONE. (2013) 8:e83138. doi: 10.1371/journal.pone.0083138

11. Bossuyt PM, Reitsma JB, Bruns DE, Gatsonis CA, Glasziou PP, Irwig L, et al. STARD 2015: an updated list of essential items for reporting diagnostic accuracy studies. Clin Chem. (2015) 61:1446-52. doi: 10.1373 /clinchem.2015.246280

12. Whiting PF, Rutjes AW, Westwood ME, Mallett S, Deeks JJ, Reitsma JB, et al. QUADAS-2: a revised tool for the quality assessment of diagnostic accuracy studies. Ann Intern Med. (2011) 155:529-36. doi: 10.7326/0003-4819-155-8-201110180-00009

13. Reitsma JB, Glas AS, Rutjes AW, Scholten RJ, Bossuyt PM, Zwinderman $\mathrm{AH}$. Bivariate analysis of sensitivity and specificity produces informative summary measures in diagnostic reviews. J Clin Epidemiol. (2005) 58:982-90. doi: 10.1016/j.jclinepi.2005.02.022

14. Lughezzani G, Maffei D, Paciotti M, Lazzeri M, Colombo P, Fasulo $\mathrm{V}$, et al. Assessing the diagnostic accuracy of micro-ultrasound for the detection of clinically significant prostate cancer: results from a single-institutional experience. Eur Urol Suppl. (2019) 18:e1883. doi: 10.1016/S1569-9056(19)31365-X

15. Astobieta Odriozola A, Sanchez A, De La Cruz I, Pereira JG, Gamarra $\mathrm{M}$, Urdaneta $\mathrm{F}$, et al. Initial results comparing $29 \mathrm{MHz}$ micro-ultrasound with multi-parametric MRI for targeted prostate biopsy: relative sensitivity to clinically significant prostate cancer. Eur Urol Suppl. (2018) 17:e901. doi: 10.1016/S1569-9056(18)31462-3

16. Abouassaly R, Klein EA, El-Shefai A, Stephenson A. Impact of using $29 \mathrm{MHz}$ high-resolution micro-ultrasound in real-time targeting of transrectal prostate biopsies: initial experience. World J Urol. (2019) doi: 10.1007/s00345-019-02863-y. [Epub ahead of print].

17. Chessa F, Schiavina R, Brunocilla E, Marco B, Bianchi L, Pultrone C, et al. Diagnostic accuracy of the novel $29 \mathrm{MHZ}$ micro-ultrasound "exactvutm" for the detection of clinically significant prostate cancer: a prospective single institutional study. J Urol. (2019) 201:E1081. doi: 10.1097/01.JU.0000557292.67482.4b

18. Claros OR, Muttin F, Tourinho-Barbosa RR, Gallardo AC, Barret E, Rozet F, et al. Comparison of cancer detection rates in micro-ultrasound biopsies Hour robotic ultrasound-magnetic resonance imaging fusion biopsies for prostate cancer. J Urol. (2019) 201:E184-5. doi: 10.1097/01.JU.0000555295.45193.66

19. Luger F, Gusenleitner A, Kaar J, Mayr C, Loidl W. A prospective validation of the diagnostic accuracy of pri-mus for prostate cancer risk identification. $J$ Urol. (2019) 201:E1078. doi: 10.1097/01.JU.0000557263.76891.fa

20. Eure G, Fanney D, Lin J, Wodlinger B, Ghai S. Comparison of conventional transrectal ultrasound, magnetic resonance imaging, and micro-ultrasound for visualizing prostate cancer in an active surveillance population: a feasibility study. Cuaj Can Urol Assoc J. (2019) 13:E70-7. doi: 10.5489/cuaj.5361

21. Cai W, Zhu D, Byanju S, Chen J, Zhang H, Wang Y, et al. Magnetic resonance spectroscopy imaging in diagnosis of suspicious prostate cancer: a metaanalysis. Medicine. (2019) 98:e14891. doi: 10.1097/MD.0000000000014891

22. van Luijtelaar A, Bomers J, Futterer J. A comparison of magnetic resonance imaging techniques used to secure biopsies in prostate cancer patients. Expert Rev Anticancer Ther. (2019) 19:705-16. doi: 10.1080/14737140.2019.1641086

23. Maggi M, Panebianco V, Mosca A, Salciccia S, Gentilucci A, Di Pierro $\mathrm{G}$, et al. Prostate imaging reporting and data system 3 category cases at multiparametric magnetic resonance for prostate cancer: a systematic review and meta-analysis. Eur Urol Focus. (2019) doi: 10.1016/j.euf.2019.06.014. [Epub ahead of print].

24. Deeks JJ, Altman DG. Diagnostic tests 4: likelihood ratios. BMJ. (2004) 329:168-9. doi: 10.1136/bmj.329.7458.168

Conflict of Interest: The authors declare that the research was conducted in the absence of any commercial or financial relationships that could be construed as a potential conflict of interest.

Copyright (c) 2019 Zhang, Wang, Wu, Jing, Chen, Zhang, Xu, Liu and Chen. This is an open-access article distributed under the terms of the Creative Commons Attribution License (CC BY). The use, distribution or reproduction in other forums is permitted, provided the original author(s) and the copyright owner(s) are credited and that the original publication in this journal is cited, in accordance with accepted academic practice. No use, distribution or reproduction is permitted which does not comply with these terms. 